Case report

\title{
Merkel cell carcinoma of the upper extremity
}

\author{
Iuliana Giorgiana Spiridon ${ }^{*}$, , Cristina Cozma ${ }^{1}$, Laura Răducu ${ }^{1,2}$, Cristian Radu \\ Jecan $^{1,2}$
}

${ }^{1}$ Department of Plastic and Reconstructive Surgery, "Prof. Dr. Agrippa Ionescu" Clinical Emergency Hospital, Bucharest, Romania; ${ }^{2 " C a r o l ~ D a v i l a " ~ U n i v e r s i t y ~ o f ~ M e d i c i n e ~ a n d ~ P h a r m a c y, ~ B u c h a r e s t, ~}$ Romania

\begin{abstract}
Merkel cell carcinoma is a rare, aggressive skin cancer which is usually characterized by lymph node involvement, distant metastases, and disease relapse. It is commonly seen in elder people and it affects the head, neck, and extremities. It can be difficult to differentiate macroscopically Merkel cell carcinoma from other small cells neoplasms. Herein is the case of a 66-year-old male patient, admitted in our department for a local recurrence of Merkel cell carcinoma on the posterolateral side of the proximal third right forearm. The surgical treatment included wide local excision with $2 \mathrm{~cm}$ safety margins, sentinel lymph node biopsy and grafting the residual defect using split-thickness skin. Postoperative no local or general complications were encountered. The final histological examination revealed Merkel cell carcinoma with all the excisional margins free of tumor and two positive sentinel lymph nodes. The patient has performed postoperative radiotherapy with monthly clinical examination. Six months after the last surgery and after undergoing radiotherapy, there were no local or distant signs of recurrence. Due to high rates of recurrence, close surveillance is demanded.
\end{abstract}

Keywords: Merkel cell carcinoma; sentinel lymph node biopsy; local recurrence; radiotherapy

\section{Introduction}

Merkel cell carcinoma (MCC) is a rare, aggressive neuroendocrine-derived cutaneous carcinoma which is usually characterized by lymph node involvement, distant metastases, and disease relapse [1]. The first description of Merkel cell carcinoma was made by Cyril Toker in 1972 [2]. Based on the histologic features, he named the tumor trabecular carcinoma [3]. It is a disease that mostly affects fair-skin elder people and is linked to

Received: May 2018; Accepted after review: August 2018; Published: September 2018.

${ }^{*}$ Corresponding author: Iuliana Giorgiana Spiridon MD, Department of Plastic and Reconstructive Surgery, "Prof. Dr. Agrippa Ionescu" Clinical Emergency Hospital, 7 Ion Mincu Street, 011356, Bucharest, Romania.

Email: iuliana.g.spiridon@gmail.com ultraviolet exposure, immunosuppression, and a recently described Merkel cell polyomavirus [4-6].

Cytokeratin-20 (CK-20) is a specific and sensitive marker for MCC that increased detection of the new cases. It is reported that the incidence of MCC has tripled over the past two decades [7].

MCC treatment requires wide excision alone in early stages, with adjuvant radiation therapy (RT) in advanced cases or recurrences. The addition of $\mathrm{RT}$ reduces the risk of recurrence and improves overall survival [8].

\section{Case report}

A 66-years-old patient presented to with a new tumor over the excisional scar performed 3 months before, in another hospital. Microscopic examination of the primary tumor 
(performed in another hospital) revealed malignant proliferation with small round cell, frequent mitosis, necrotic hemorrhagic areas and associated chronic inflammation. The tumor proliferation concerns adjacent fibroadipose tissue. Histological appearance suggests small cell undifferentiated Merkel Cell carcinoma, confirmed through IHC with positive values of approximately $80 \%$ for Ki67, diffusely positive values for Synaptophysin,
Chromogranin A and CK20 in tumor cells with a paranuclear dot-like granule.

Local examination revealed firm, redviolet, rapid growing and painless, smooth surface cutaneous nodule on the posterolateral side of the proximal third right forearm of $3.5 / 2.5 / 0.9 \mathrm{~cm}$ (Figure 1). There was no evidence of palpable lymph nodes. Patient's medical history was insulin-treated type 2 Diabetes mellitus and arterial hypertension.

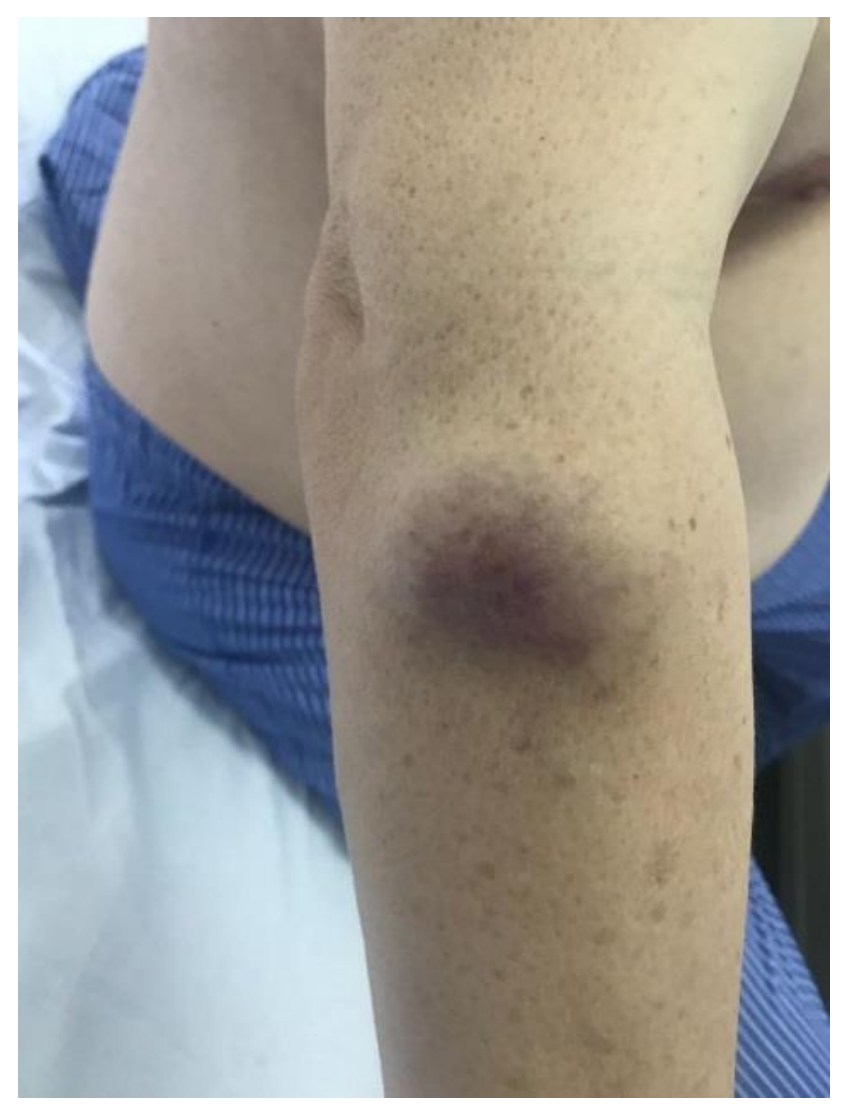

Fig. 1. The tumor aspect at patient presentation

CT scan for distant metastasis did not detect any suspicious lesions.

The magnetic resonance image (MRI) with intravenous contrast showed intense gadolinophilic mass with a small area of central necrosis, developed in the posterolateral side of the proximal third right forearm, infiltrating into the neighboring subcutaneous fat and possibly into the fascia of the extensor muscles of the carp and fingers, apparently without invading the muscle fibers. Recommended positron emission tomography was not performed because it was not covered by the patient's health care insurance.

Considering the aspect and the size of the tumor, surgical treatment wide local excision was performed under general anesthesia. The skin was incised circumferentially at $2 \mathrm{~cm}$ from the macroscopic margins of the tumor in the subfascial plane, including the initial postoperative scar (Figure 2). Intraoperatively, sentinel lymph node (SLN) was performed with peritumoral injection of methylene blue (Figure $3)$. 


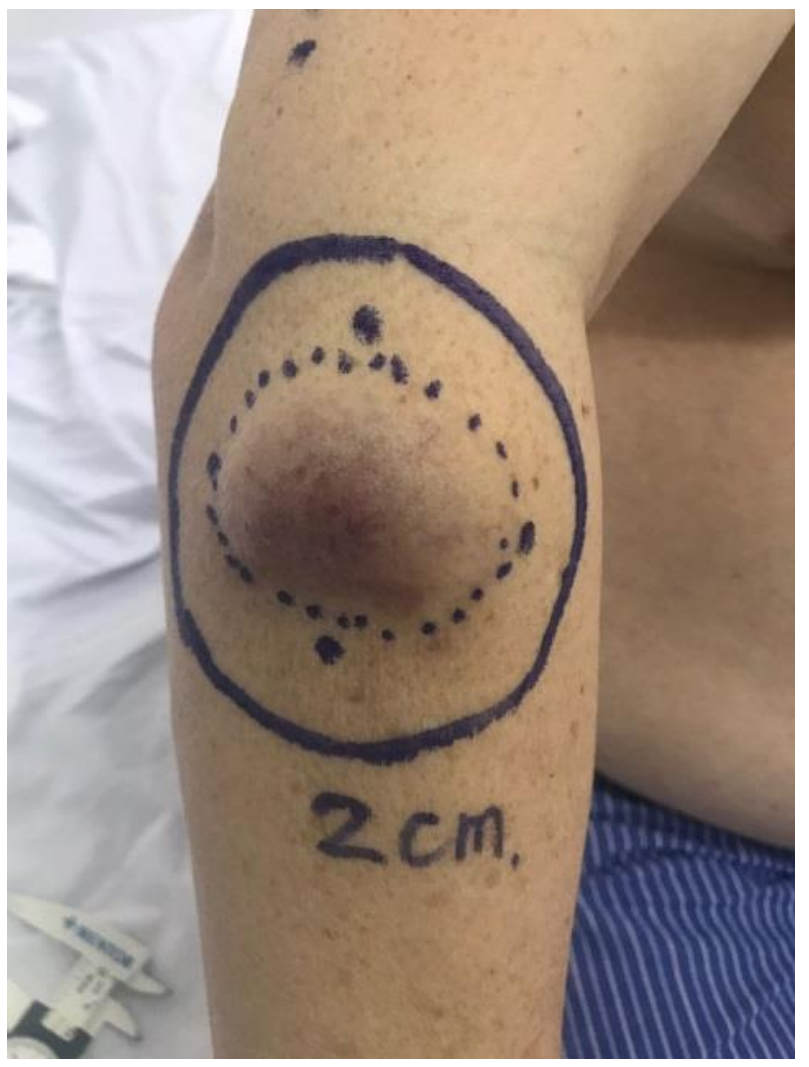

Fig. 2. Preoperative skin marks with $2 \mathrm{~cm}$ safety margins from tumor edges

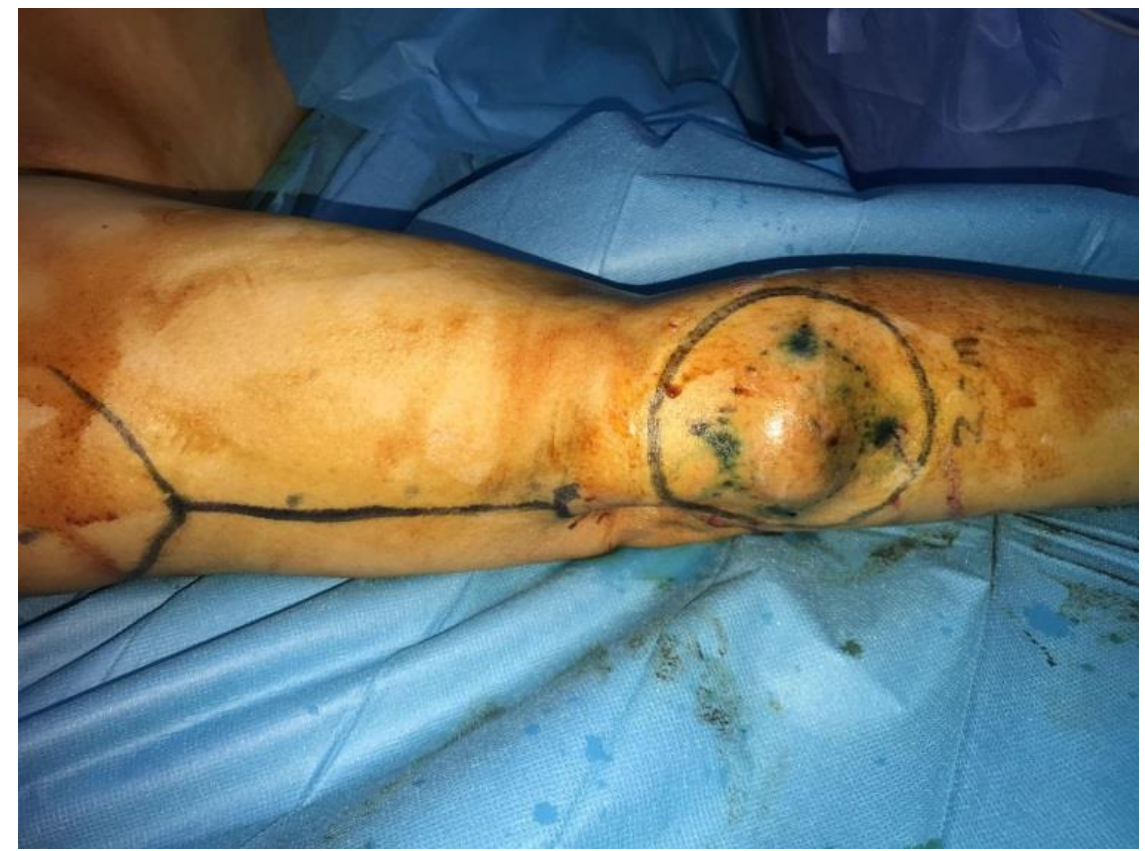

Fig. 3. Intraoperative aspect after peritumoral injection of methylene blue in four quadrants

Two lymph nodes that were infiltrated with methylene blue were extracted and evaluated histologically.
The post-excision defect of $5.5 / 4.5 \mathrm{~cm}$ was grafted using split thickness skin harvested from anterolateral right thigh and the graft was fixed with metal staples (Figures 4 and 5). 


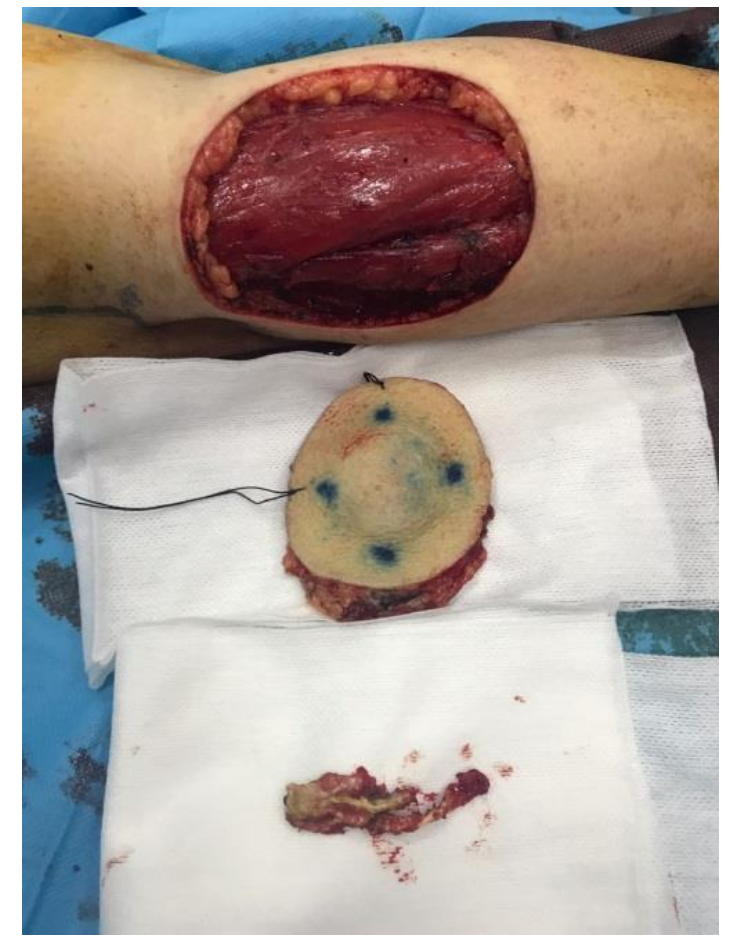

Fig. 4. The defect resulted after excision (top) and the specimen (bottom)

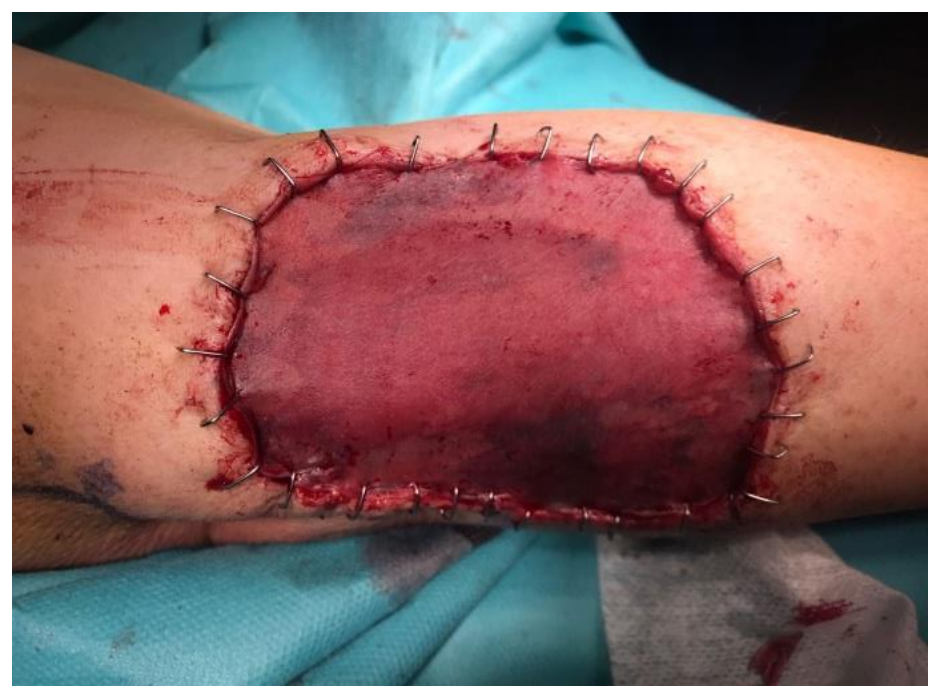

Fig. 5. Immediate postoperative aspect: the defect was grafted with split thickness skin

The postoperative recovery of the patient was mainly uneventful. He was discharged 7 days after the surgery with split-thickness skin graft fully integrated.

Final histological examination was significant for MCC with tumor free margins. The circumferential resection margin from pathology report was $20 \mathrm{~mm}$ and the deep margin was $3 \mathrm{~mm}$. Microscopic examination revealed lympho-vascular invasion, a nonbrisk lymphocytic infiltrate, mitotic rates > $1 / \mathrm{mm} \mathrm{2,} \mathrm{with} \mathrm{extension} \mathrm{of} \mathrm{the} \mathrm{tumor} \mathrm{to} \mathrm{the}$ muscle fascia and a nodular tumor growth pattern. The exam also showed evidence of metastatic disease for two SLNs.

The multidisciplinary tumor committee, which included an oncologist, radiotherapist and surgeon, recommended loco-regional adjuvant radiotherapy with constant follow-up. Radiotherapy was started one month after surgery on the tumor site and in the axilla with a total dose of 60 Gray. Six months after the last surgery and after undergoing loco-regional radiotherapy, there were no local or distant signs of recurrence (Figure 6). 


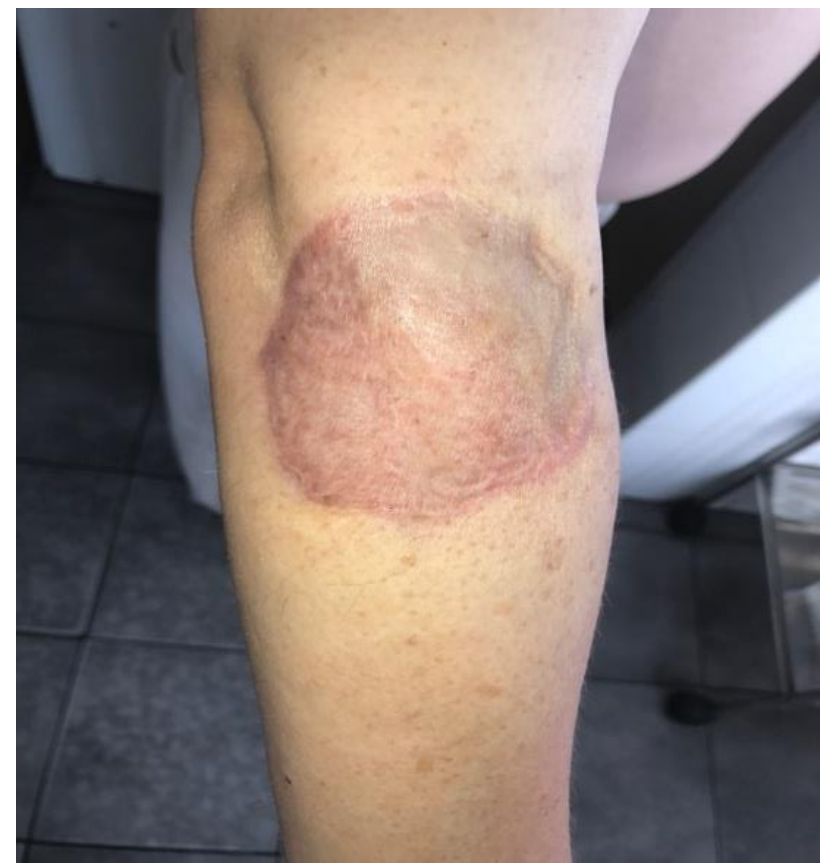

Fig. 6. Postoperative at 6 months - skin graft integrated, with no signs of local recurrence

\section{Discussions}

Merkel's cell carcinoma MCC is a rare neuroendocrine skin cancer with high probability of relapse and associated mortality developing mainly on the sun-exposed skin, namely in the head and neck region, followed by the extremities and the trunk [7, 9]. MCC incidence shows a progressive increase with age. There is a small number of cases in patients aged less than 50 years, and the average age at diagnosis is around 65 years [10]. Incidence is slightly more elevated in male than female patients and notably higher in fair-skin than darker tones skin people [11, 12].

According to a large number of studies, local recurrence occurs in $25 \%$ to $30 \%$ of all cases of MCC, regional recurrence in $52 \%$ to $59 \%$ of cases and metastatic disease in $34 \%$ to $36 \%$ of all cases. Overall, a 5 - year survival rates for MCC is specific to a scale between $30 \%$ and $64 \%$ of the cases $[13,14]$. The MCC mortality rate surpasses that of melanoma [1517].

The diagnosis of MCC is frequently not clinically suspected. Primary workup for a suspicious lesion begins with a complete exam of the skin and lymph nodes followed by biopsy. Histological examination is required before treatment planning for recurrence prognosis and long-term results. Concerning the histopathology examination, MCC is similar to a variety of other small cell tumors, hence the necessity of confirmation through IHC staining. Cytokeratin-20 (CK20) appears in approximately $95 \%$ of Merkel cell carcinoma, typically in a paranuclear dot pattern and it helps distinguish it from morphologically similar entities including metastatic small cell lung carcinoma [18-21]. Other IHC markers such as Thyroid transcription factor 1, CD56, Synaptophysin, Chromogranin $\mathrm{A}$ and neuro-filament protein can be used to exclude other diagnosis considerations [22].

Management of this highly aggressive tumor requires multidisciplinary approach to achieve best treatment [23]. Surgery remains the mainstay of MCC treatment. Wide local excision with 1 to $2 \mathrm{~cm}$ margins to investing fascia layer is the standard surgical technique [24]. Mohs surgery or complete circumferential peripheral and deep-margin assessment (CCPDMA) are recommended where cosmetic results are critical. A study of 661 cases published by Tai et al. showed that complete excision is linked to significant overall survival 
improvement. Before reconstruction involving extensive undermining or tissue movements, negative histological margins are required. For cases where primary wound closure is not possible, split-thickness skin graft proves as a good option to monitor relapse [25]. The definitive surgery may alter lymphatic drainage; therefore coordinated treatment recommends sentinel lymph node biopsy (SLNB) prior to surgery.

In many centers, SLNB has become the standard instrument in the nodal staging of MCC. There are multiple reviews which document the prognostic importance of positive SLN in MCC [26-28]. Many studies reported that the recurrence rates of the patients with positive SLN are three times more significant and the disease related mortality is two times higher compared to negative SLN patients $[29,30]$. A large retrospective analysis of prognostic factors of 5,823 patients with MCC showed that the pathology evaluation of node involvement significantly improved predictability of survival rates compared to clinical or radiologic examination [14]. Nevertheless, the literature is not unanimous in this regard and some authors did not find significant correlation between positive SLN pathology and relapse or survival rates [31]. SLNB has been recommended as a standard procedure for disease staging in patients with MCC in the NCCN guidelines [32]. The most important considerations in staging MCC are tumor size (greater or less than $2 \mathrm{~cm}$ ), the invasion of the underlying structures, the lymph node involvement, with a differentiation between either clinical or pathological node status and the presence of metastasis [33].

Herein patient was staged as IIIA, pT4, N1a, M0 according to the 8th AJCC consensus guidelines for MCC [34]. This stage of disease (microscopic nodal) is frequently detected via SLNB, and the recommended treatment includes radiotherapy or completion lymph node dissection (CLND). In our case, radiotherapy was achieved. A study by Fang et al which analyzed the outcomes of patients with lymph node-positive MCC treated with lymph node irradiation as definitive therapy compared with CLND reported no significant difference with regard to overall survival. Both microscopic and palpable lymph node disease registered a great regional control rate (comparable to CLND) through lymph node irradiation applied to positive lymph node [28]. The use of adjuvant radiation therapy after complete excision lowered the risk of recurrences. It is reported a large analysis of 1,187 cases that demonstrated a higher overall survival for patients undergoing adjuvant RT compared to those who did not received RT. In a series of 83 cases, patients revealed no overall survival improvement with adjuvant RT; however regional recurrence has registered considerably risk reduction in comparison with the observational group [8]. For our patient, the decision was for locoregional radiation therapy, due to aggressive features and high risk of tumor recurrence. Close follow-up with complete clinical examination monthly in the first 3 to 6 months, every 3 to 6 months for the first 2 years, then every 6 to 12 months thereafter. Routine imaging ought to be performed for high-risk patients to identify distant metastases. Selfexamination of the skin is also useful, MCC patients show greater risk for other nonmelanoma skin cancers.

\section{Conclusion}

The management of MCC, which is a rare but very aggressive skin cancer, is a complex activity that requires a multidisciplinary approach. Wide local excision of the local recurrence and SLNB removed the tumor and identified the nodal spreading. Local and regional adjuvant radiotherapy afforded no recurrence at short-term follow-up. However, due to high rates of relapse, clinical and imagistic surveillance is required to evaluate long-term results.

\section{Consent}

Written informed consent was obtained from the patient for publication of this case report.

\section{Competing interests}

The authors declare that they have no competing interests. 


\section{References}

1. Becker JC. Merkel cell carcinoma. Ann Oncol 2010; 21(Suppl 7):81- 85.

2. Kim JA, Choi AH. Effect of radiation therapy on survival in patients with resected Merkel cell carcinoma: a propensity score surveillance, epidemiology, and end results database analysis. JAMA Dermatol 2013; 149(7):831838.

3. Toker C. Trabecular carcinoma of the skin. Arch Dermatol 1972; 105(1):107-110.

4. Engels EA, Frisch M, Goedert JJ, Biggar RJ,Miller RW. Merkel cell carcinoma and HIV infection. Lancet 2002; 359:497-498.

5. Feng $H$, Shuda $M$, Chang $Y$, Moore PS. Clonal integration of a polyomavirus in human Merkel cell carcinoma. Science 2008; 319:1096-1100.

6. Penn I, First MR. Merkel's cell carcinoma in organ recipients: report of 41 cases. Transplantation 1999; 68:1717-1721.

7. Heath $M$, Jaimes $N$, Lemos $B$, et al. Clinical characteristics of Merkel cell carcinoma at diagnosis in 195 patients: the AEIOU features. J Am Acad Dermatol 2008; 58:375-381.

8. Mojica $P$, Smith D, Ellenhorn JD. Adjuvant radiation therapy is associated with improved survival in Merkel cell carcinoma of the skin. $J$ Clin Oncol 2007; 25:1043-1047.

9. Coggshall K, Tello TL, North JP, Yu SS. Merkel cell carcinoma: An update and review: Pathogenesis, diagnosis, and staging. $J A m$ Acad Dermatol 2018; 78:433-442.

10. Agelli M, Clegg LX. Epidemiology of primary Merkel cell carcinoma in the United States [abstract]. J Am Acad Dermatol 2003; 49(5):832-841.

11. Hodgson NC. Merkel cell carcinoma: changing incidence trends. J Surg Oncol 2005; 89(1):1-4.

12. Albores-Saavedra J, Batich $K$, Chable-Montero F, Sagy N, Schwartz AM, Henson DE. Merkel cell carcinoma demographics, morphology, and survival based on 3870 cases: a population based study. J Cutan Pathol 2010; 37:20-27.

13. Bichakjian CK, Lowe L, Lao CD, et al. Merkel cell carcinoma: critical review with guidelines for multidisciplinary management. Cancer 2007; 110:1-12.

14. Lemos BD, Storer BE, lyer JG, et al. Pathologic nodal evaluation improves prognostic accuracy in Merkel cell carcinoma: analysis of 5823 cases as the basis of the first consensus staging system. J Am Acad Dermatol 2010; 63(5):751-761.

15. Akhtar S, Oza KK, Wright J. Merkel cell carcinoma: report of 10 cases and review of the literature. J Am Acad Dermatol 2000; 43:755767.

16. Gillenwater AM, Hessel AC, Morrison WH, et al. Merkel cell carcinoma of the head and neck: effect of surgical excision and radiation on recurrence and survival. Arch Otolaryngol Head Neck Surg 2001; 127:149-154.

17. Medina-Franco $\mathrm{H}$, Urist MM, Fiveash J, Heslin MJ, Bland $\mathrm{KI}$, Beenken SW. Multimodality treatment of Merkel cell carcinoma: case series and literature review of 1024 cases. Ann Surg Oncol 2001; 8:204-208.

18. Bobos M, Hytiroglou $P$, Kostopoulos I, Karkavelas G, Papadimitriou CS. Immunohistochemical distinction between merkel cell carcinoma and small cell carcinoma of the lung. Am J Dermatopathol 2006; 28:99104.

19. Hanly AJ, Elgart GW, Jorda M, Smith J, Nadji M. Analysis of thyroid transcription factor- 1 and cytokeratin 20 separates Merkel cell carcinoma from small cell carcinoma of lung. J Cutan Pathol 2000; 27:118-120.

20. Scott MP, Helm KF. Cytokeratin 20: a marker for diagnosing Merkel cell carcinoma. Am J Dermatopathol 1999; 21:16-20.

21. Harms PW, Collie AM, Hovelson $\mathrm{DH}$, et al. Next generation sequencing of cytokeratin 20negative Merkel cell carcinoma reveals ultraviolet signature mutations and recurrent TP53 and RB1 inactivation. Mod Pathol 2016; 29(3):240-248.

22. Gruber SB, Wilson LD. Merkel cell carcinoma. Cutaneous Oncology: pathophysiology, diagnosis, and management. Science; 1998:710-721.

23. Schneider S, Thurnher D, Erovic BM. Merkel cell carcinoma: interdisciplinary management of a rare disease. J Skin Cancer 2013; 2013:189342.

24. Tai P. A practical update of surgical management of Merkel cell carcinoma of the skin. ISRN Surg 2013; 2013:850797.

25. Tai PT, Yu E, Tonita J, Gilchrist J. Merkel cell carcinoma of the skin. J Cutan Med Surg 2000; 4:186-195.

26. Howle JR, Hughes TM, Gebski V, Veness MJ. Merkel cell carcinoma: an Australian perspective and the importance of addressing the regional lymph nodes in clinically nodenegative patients. J Am Acad Dermatol 2012; 67:33-40.

27. Fields RC, Busam KJ, Chou JF, et al. Recurrence and survival in patients undergoing sentinel lymph node biopsy for Merkel cell 
carcinoma: analysis of 153 patients from a single institution. Ann Surg Oncol 2011; 18:2529-2537.

28. Fang LC, Lemos B, Douglas J, lyer J, Nghiem P. Radiation monotherapy as regional treatment for lymph node-positive Merkel cell carcinoma. Cancer 2010; 116:1783-1790.

29. Gupta SG, Wang LC, Peñas PF, Gellenthin M, Lee SJ, Nghiem P. Sentinel lymph node biopsy for evaluation and treatment of patients with Merkel cell carcinoma: the Dana-Farber experience and meta-analysis of the literature. Arch Dermatol 2006; 142(6):685-690.

30. Mehrany K, Otley CC, Weenig RH, Kim Phillips P, Roenigk RK, Nguyen TH. A meta-analysis of the prognostic significance of sentinel lymph node status in Merkel cell carcinoma. Dermatol Surg 2002; 28:113-117.
31. Smith VA, Camp ER, Lentsch EJ. Merkel cell carcinoma: identification of prognostic factors unique to tumors located in the head and neck based on analysis of SEER data. Laryngoscope 2012; 122:1283-1290.

32. Bichakjian CK, Olencki T, Aasi S, et al. NCCN clinical practice guidelines in oncology (NCCN guidelines) Merkel cell carcinoma, version 1 2018. J Natl Compr Canc Netw 2018; 16(6):742-774.

33. Asgari MM, Sokil MM, Warton EM, lyer J, Paulson KG, Nghiem P. Effect of host, tumor, diagnostic, and treatment variables on outcomes in a large cohort with Merkel cell carcinoma. JAMA Dermatol 2014; 150:716723.

34. Amin MB, Edge SB, Greene FL, et al. AJCC Cancer Staging Manual, $8^{\text {th }}$ Edition. New York: Springer, 2017. 